the spatial isolation of poor people in cities (the "spatial poverty trap") as one of the major challenges in developing countries. As people and cities in the developing world get richer, the worry is that the spatial socioeconomic segregation of poor people increases, which in turn may increase their risks of mortality and poor health. Methods Data from 15 major Brazilian cities were analysed, with spatial measures of socioeconomic segregation ("isolation of the poor") estimated for Brazilian districts within cities. The association of the spatial isolation of the poor with district level mortality rates was examined using multiple membership multilevel Poisson regression models to take account of the multilevel (districts within cities) and spatial nature of the data.

Results Increasing spatial isolation of the poor tends to be associated with higher mortality rates, with an interaction between income and spatial isolation. There is not much difference in mortality rates among the poorest districts in terms of spatial isolation. However in the richest districts, districts where the poor are spatially isolated have the highest mortality rates, whereas districts where the poor are not isolated have the lowest mortality rates.

Conclusion As cities in the developing world get richer, there is a risk that this leads to increasing spatial socioeconomic segregation of the poor within those cities. The results from this study suggests that the spatial dimension of poverty within cities may be just as important to health as poverty levels.

\section{OP04 THE ENGLISH NORTH-SOUTH DIVIDE: RISK FACTORS FOR CARDIOVASCULAR DISEASE ACCOUNTING FOR CROSS- SECTIONAL SOCIOECONOMIC-POSITION}

doi:10.1136/jech-2012-201753.004

R Kumar, ARH Dalton. Primary Care \& Public Health, Imperial College London, London, UK

Background Geographic inequalities in health are widely discussed, with an English North-South divide a popular notion. Data indicate the North-South divide in all cause mortality has persisted, even widening in recent years. Given the impact of cardiovascular disease (CVD) mortality on health inequalities, we aimed to assess the extent of a salient North-South divide in risk factors for CVD, controlling for markers of socioeconomic-position (SEP).

Methods We conducted a cross-sectional analysis using the 2006 Health Survey for England using respondents aged 16 years and over. We assessed the population means of systolic blood pressure, total cholesterol, body mass index (BMI) and smoking prevalence. We built nested regression models (all linear regression except for logistic for smoking) adding; demographic (age /sex /ethnicity), SEP indicators (individual income, education attainment, housing tenure, car ownership, occupational classification and area level IMD), behavioural risk factors and vascular disease status. We tested variables for multi-collinearity, assumptions of normality for linear outcomes and use valid survey weights. We finally examine interactions between the North-South divide and age and sex on the risk factors.

Results The North of England showed more deprived characteristics across markers of SEP; except for greater home-ownership. Controlling for demographic variables, we found a significant North-South difference (excess in North) in systolic blood pressure (1.94 [se=0.38]), BMI (0.47 [0.11]) and smoking prevalence $(2.93 \%$ [0.50]). The difference in smoking prevalence was entirely abolished by markers of SEP; both systolic blood pressure and BMI differences were attenuated by SEP, behavioural and vascular disease indicators (1.52 [0.38] \& 0.31 [0.11] respectively), but remained significant. The North-South divide in systolic blood pressure was attributed to differences in men (2.18 [0.54]), being non-significant in women; and in middle age groups (2.70 [0.76] aged 40-59, compared with 2.29 [0.78] aged 16-39) and was non-significant aged 60 and over.
Conclusion Smoking is a major factor behind morbidity and mortality. In line with work from different settings, patterns in smoking can be explained through adverse, cross-sectional patterns of SEP. Addressing underlying poverty and disadvantage may be required to fully tackle smoking inequalities. Using a suite of measures designed to address different constructs of SEP, although cross-sectional, we find excesses in blood pressure and BMI in the North of England. These differences may, in part, explain previously found differences in mortality. If we are to understand, and therefore reduce, geographic inequalities, current measures of SEP may require improvement, for example accounting for aspects of the life-course.

\section{Public Health Interventions: Area and Weight Management}

\section{OP05 FROM TRIAL TO POPULATION: EFFECT OF A WEIGHT MANAGEMENT INTERVENTION ON BODY MASS INDEX WHEN SCALED UP}

doi:10.1136/jech-2012-201753.005

1J Fagg, ${ }^{1 T}$ Cole, ${ }^{2} \mathrm{~S}$ Cummins, ${ }^{1} \mathrm{H}$ Goldstein, ${ }^{3} \mathrm{H}$ Roberts, ${ }^{1} \mathrm{C}$ Law. ${ }^{1} \mathrm{M} R \mathrm{C}$ Centre of Epidemiology for Child Health , UCL Institute of Child Health, London, UK; ${ }^{2}$ Department of Geography, Queen Mary, University of London, London, UK; ${ }^{3}$ General and Adolescent Paediatrics Unit, UCL Institute of Child Health, London, UK

Background The evidence base for effective interventions to manage childhood overweight and obesity is growing. However, results from research may not generalise to service delivery settings, and scaled-up interventions may not reduce health inequalities. We examine a scaled-up childhood weight management intervention, examining variations in body mass index by person, family, place and programme

Methods MEND 7-13 (Mind, Exercise, Nutrition, Do It!) is a multi-component family-based community weight management intervention shown to be effective in a randomised controlled trial (RCT). We used MEND service data from 10,080 children attending MEND programmes from 2007-10. Outcomes were: Change in Body Mass Index (BMI) change over the programme (10 weeks). Anthropometry is measured by trained staff following standardised procedures. We estimated associations between BMI change and participant, family, neighbourhood and programme factors - including pre-specified interactions and random slopes. Multilevel multivariate regression models were used with multiple imputation for missing values. We described participants by BMI at programme start (pre-BMI), age, sex and ethnicity; families by parent-reported employment, lone parent and housing tenure status; places by residential neighbourhood income deprivation, urbanicity, food and built environments; and programmes by the percentage of sessions attended and variables describing programme composition (e.g. group size)

Results BMI was reduced by similar amounts in the RCT and service delivery (RCT BMI change $=-0.91$ [95\%CI: -1.13 to $-0.68]$ ), service BMI change $=-0.75[-0.78$ to -0.73$]$. Service BMI reductions were clinically significant in all socio-demographic groups analysed. However, in multilevel models, pre-BMI, age, ethnicity, unemployment status and programme attendance were independently associated with BMI change. For example, in comparison to white children, reductions in BMI were statistically significantly smaller for black and minority ethnic group children (Asian b=0.29, $\mathrm{p}<0.001$, Black $b=0.20, p<0.001$, (positive coefficients indicate smaller BMI reduction)). Similarly, BMI fell less in children whose parents were unemployed $(b=0.14, p<0.001$, baseline employed parent). There were no significant differences by sex or neighbourhood factors. Tests of interactions and random slopes were non-significant. 
Conclusion Clinically significant BMI changes, similar to those achieved under research conditions, may be replicable in service delivery settings for children of all socio-demographic groups analysed. However, at the population level, scaled up programmes may work better for some groups than others. Public health implications of these results for health inequalities will be discussed.

\section{OP06 WELL LONDON: RESULTS OF A CLUSTER-RANDOMISED TRIAL OF A COMMUNITY DEVELOPMENT APPROACH TO IMPROVING HEALTH BEHAVIOURS AND MENTAL WELLBEING IN DEPRIVED INNER-CITY NEIGHBOURHOODS}

doi:10.1136/jech-2012-201753.006

${ }^{1} \mathrm{G}$ Phillips, ${ }^{2} \mathrm{R}$ Hayes, ${ }^{2} \mathrm{C}$ Bottomley, ${ }^{3} \mathrm{M}$ Petticrew, ${ }^{1} \mathrm{P}$ Watts, ${ }^{3 \mathrm{~K}}$ Lock, ${ }^{4} \mathrm{~A}$ Clow, ${ }^{5} \mathrm{~A}$ Draper, ${ }^{6} \mathrm{D}$ Moore, ${ }^{7} \mathrm{E}$ Schmidt, ${ }^{1} \mathrm{P}$ Tobi, ${ }^{1} \mathrm{~S}$ Lais, ${ }^{1} \mathrm{G}$ Yu, ' $\mathrm{G}$ Barrow-Guevara, ${ }^{1} \mathrm{~A}$ Renton. ${ }^{\prime} / n s t i t u t e$ for Health and Human Development, University of East London, London, UK; ${ }^{2}$ Faculty of Epidemiology and Population Health, London School of Hygiene and Tropical Medicine (LSHTM), London, UK; ${ }^{3}$ Faculty of Public Health and Policy, LSHTM, London, UK; ${ }^{4} \mathrm{De}-$ partment of Psychology, University of Westminster, London, UK; ${ }^{5}$ Department of Human and Health Sciences, University of Westminster, London, UK; ${ }^{\circ} / n s t i t u t e$ for Research on Child Development, University of East London, London, UK

Background Few public health interventions combining modification of the social and built environment with individual-level health promotion have been robustly evaluated in the UK. Well London is an assets-based community development programme designed to improve physical activity, healthy eating and mental wellbeing in highly deprived inner-city communities. The programme, delivered between 2007 and 2011, comprised a mix of projects delivering traditional health promotion, community development and changes to the physical neighbourhood environment. The objectives of the study are to: (i) determine the effectiveness of Well London for improving healthy eating, physical activity and mental wellbeing in deprived inner-city communities; (ii) examine the effects in population subgroups linked to health inequalities in the UK.

Methods We used a pair-matched, cluster-randomised trial with 20 control neighbourhoods matched within London boroughs to 20 programme delivery neighbourhoods. The trial outcomes in adult residents (aged $\geq 16$ years) were collected using a structured electronic household survey, administered by fieldworkers to 100 randomly sampled residents in each intervention and control neighbourhood. The main outcome measures were: physical activity: meeting UK Chief Medical Officer-recommended five sessions of 30 minutes moderate intensity activity per week (selfreport International Physical Activity Questionnaire); healthy eating: eating at least five portions of fruit/vegetables per day (food frequency questionnaire from the Health Survey for England); and mental wellbeing: abnormal score on 12-item General Health Questionnaire; Warwick Edinburgh Mental Wellbeing Scale score.

Results The baseline survey in 2008 showed that the intervention and control populations are comparable on socio-demographic/economic characteristics and primary trial outcomes. At baseline, $37 \%$ of adults met the five-a-day (healthy eating), $60 \%$ met the five-aweek (physical activity), and $18 \%$ reported experiencing anxiety or depression. Results from the follow-up survey will be available in April 2012. We will present the effects of Well London on the primary outcomes and subgroup analyses by gender, age, ethnicity and level of education.

Conclusion In a health system where less than $1 \%$ of the research budget is spent on primary preventive interventions for non-communicable diseases, robust evidence about the effectiveness and cost-effectiveness of upstream interventions is essential for action on health inequalities and reductions in healthcare spending recommended by the Marmot Review (2010) and the Wanless report (2004).

\section{OP07 WHAT SHAPES PARTICIPATION IN A COMMUNITY- BASED INTERVENTION? EVIDENCE FROM A QUALITATIVE EVALUATION OF THE WELL LONDON PROJECT}

doi:10.1136/jech-2012-201753.007

${ }^{1} \mathrm{~S}$ Jain, ${ }^{2} \mathrm{~A}$ Draper, ${ }^{3} \mathrm{~A}$ Clow, ${ }^{4} \mathrm{R}$ Lynch, ${ }^{2} \mathrm{~J}$ Derges. ${ }^{1}$ Social Work Subject Area, School of Social and Political Science, University of Edinburgh, Edinburgh, UK; ${ }^{2}$ Department of Human and Health Sciences, University of Westminster, London, UK; ${ }^{3}$ Department of Psychology, University of Westminster, London, UK; ${ }^{4}$ Department of Anthropology, UCL, London, UK

Background This paper examines how individual and area-level contextual factors shape participation in a community-based development and health promotion intervention. Well London was a 3 -year community development and health promotion programme for improving health behaviours (physical activity and healthy eating) and mental health and wellbeing in areas of high deprivation. The programme aimed to improve individual level health outcomes through a combination of neighbourhood and individual level interventions. Community engagement/participation was a central strategy of these interventions.

Methods A quantitative cluster randomised trial (CRT) was used to evaluate Well London in 20 neighbourhoods defined as Census Lower Super Output Areas (LSOAs). A qualitative study was nested within the trial to examine mechanisms and complexity. This study employed critical case sampling to select three intervention LSOAs that reflected a range of pre-existing community engagement and activities. In-depth semi-structured interviews were conducted with 59 respondents purposively sampled from each of 3 distinct areas. Each area reflected differences in implementation, nature of community life, and pre-existing community activities. Interviews addressed three topics: experiences of area, individual health \& wellbeing, and knowledge of and involvement in Well London. Transcripts were coded and thematic analysis undertaken using NVIVO software.

Results Analysis found that area level and individual-level characteristics interacted to shape specific models of individual participation in each area. In an area with a 'dispersed' community, limited pre-existing activities and implementation through formal institutions, participation was attributed by respondents to self-motivation and responses to deprivation. In contrast, in the 2 nd area, Well London implementation centred on an individual community organizer operating in a geographically close-knit area. Strong community interest and participation was shaped by the ability of this individual to inspire a sense of change. Finally, in an area with a 'saturation' of pre-existing activities, participation in Well London was part of a socially accepted pattern of community involvement. For new people to the area, involvement was viewed as aiding integration while for long-standing residents this was seen as a strategy to contribute to community life.

Conclusion Recent reviews on community participation present evidence of a causal link between participation and positive health outcomes. However, the mechanisms underlying this are not clear. The reasons people participate in Well London are shaped by interactions between individual and area-level factors. This suggests that understanding the link between community participation and health outcomes requires a contextualized analysis of why people participate and the meanings they associate with this.

\section{OP08 EVALUATING THE HEALTH INEQUALITIES IMPACT OF THE NEW DEAL FOR COMMUNITIES INITIATIVE}

doi:10.1136/jech-2012-201753.008

1J Popay, ${ }^{2} \mathrm{M}$ Whitehead, ${ }^{3} \mathrm{H}$ Badland, ${ }^{4} \mathrm{M}$ Stafford, ${ }^{5} \mathrm{~J}$ Nazroo, ${ }^{6} \mathrm{C}$ Dibbens, ${ }^{1} \mathrm{E}$ Halliday, ${ }^{2} S$ Povall. 'Division of Health Research, University of Lancaster, Lancaster, UK;'2Institute of Health, Psychology and Society, University of Liverpool, Liverpool, UK; ${ }^{3}$ Centre for Physical Activity and Nutrition, Auckland University of Technology, Auckland, Australia; 